\title{
Three Dimensional Modelling and Numerical Analysis of Super-Radiant Harmonic Emission in FEL (Optical Klystron)*
}

\author{
A. Gover, A. Friedman, A. Luccio \\ National Synchrotron Light Source \\ Brookhaven National Laboratory, Upton N. Y.
}

BNL- -38939

\section{Abstract}

DE87 003208

A full 3-D Analysis of super-radiant (bunched electron) free electron harmonic radiation is presented. A generalized form of the FEL pendulum equation was derived and numerically solved. Both spectral and phasor formulation were developed to treat the radiation in the time domain. In space the radiation field is expanded in terms of either a set of free space discrete modes or plane waves. The numerical solutions reveal some new distinctly 3-D effects to which we provide a physical explanation.

\section{Introduction}

The subject of this paper is super-radiant emission of radiation from undulated electrons in either a uniform wiggler (FEL structure) or in an optical klystron structure. The super-radiant emission scheme is a method for producing temporally and spatially coherent undulator radiation without necessarily involving any stimulated emission of radiation (lasing) [1]. It thus enables to substantially increase the spectral brightness of incoherent undulator harmonic radiation, though the total radiative power emitted by the beam is not different than in the incoherent undulator emission. It produces a less bright radiation beam than an FEL in which not only the coherence of the radiation is high, but also the total power extracted from the beam is substantially enhanced (by the stimulated emission process). However, contrary to an FEL no oscillation threshold condition needs to be attained in order to obtain super-radiant coherent harmonic emission, and consequently this scheme may be useful at short wavelengths (V.U.V.) where the FEL oscillation condition is hard to satisfy. For obtaining super-radiance, some means for bunching the electron beam

* Research carried out under the auspices of the U.S. Department of Energy under contract No. DE-AC-02-76CH00016 
must be provided in order to make the electrons emit radiation in phase with each other. These means is usually a high intensity external laser which illuminates the electrons in the undulator at the fundamental or one of the low order undulator harmonic frequencies. Alternatively, the bunching means can be an FEL which operates with the same undulator at the fundamental or one of the lower order undulator harmonic frequencies.

Experimental study of super-radiant harmonic radiation was only reported by a French research group based on an optical klystron structure [2]. In many of the recently reported FEL experiments (in all of which lasing took place in the fundamental harmonic frequency) also intense harmonic emission was observed $[3,4]$. We assert that this harmonic emission was probably super-radiant and consequently coherent. However, the features of this radiation were not studied in these experiments. An experimental program is under way in Brookhaven National Laboratory, National Synchrotron Light Source where super radiant harmonic emission is intended to be studied using a uniform wiggler (FEL) structure and an external modulating laser. This experiment is the main motivation and main model for the present theoretical analysis.

To clarify the definitions used in this article we delineate the distinction between the three different kinds of undulator based radiation schemes (spontaneous undulator radiation, super-radiant emission and lasing) by comparatively listing their definitions in Table 1. We illustrate the two main configurations of super-radiant radiators in Fig. 1a,b, and in Table 2 we list the frequency and linewidth parameters of the various radiation fields involved.

Fig. 1a depicts the optical klystron super-radiant emission scheme which was used in Orsay[2]. This configuration consists of three sections. In the first "modulation" section energy (velocity) modulation of the electron beam takes place when the beam is synchronized with the ponderomotive force of the modulating laser beam, which is radiating at frequency $\omega_{m}$ near the line center frequency of one of the low (m-th) order undulatorharmonic frequencies (for example the fundamental $-m=1$ harmonic). The laser is assumed to have a coherence time or pulse duration $\left(T_{\text {laser }}\right)$ which is larger and fully overlaps the electron beam pulse (of $T_{p u l s e}$ duration). The electrons then pass through the second dispersive section, where their energy modulation turns into phase (or density or current) modulation (bunching) at the frequency of the modulating laser and its harmonics (n). In the last "radiation" section the bunched electrons pass again through an undulator where they emit their undulator synchrotron radiation in phase with each other (superradiant emission). As is shown in the third row of Table 2 , the super-radiant frequencies are at the nm-th harmonic frequencies of the fundamental undulator frequency $\omega_{u}$. The incoherent undulator radiation (fourth row) occurs at all odd harmonics $(l=1,3,5, \ldots)$ of 


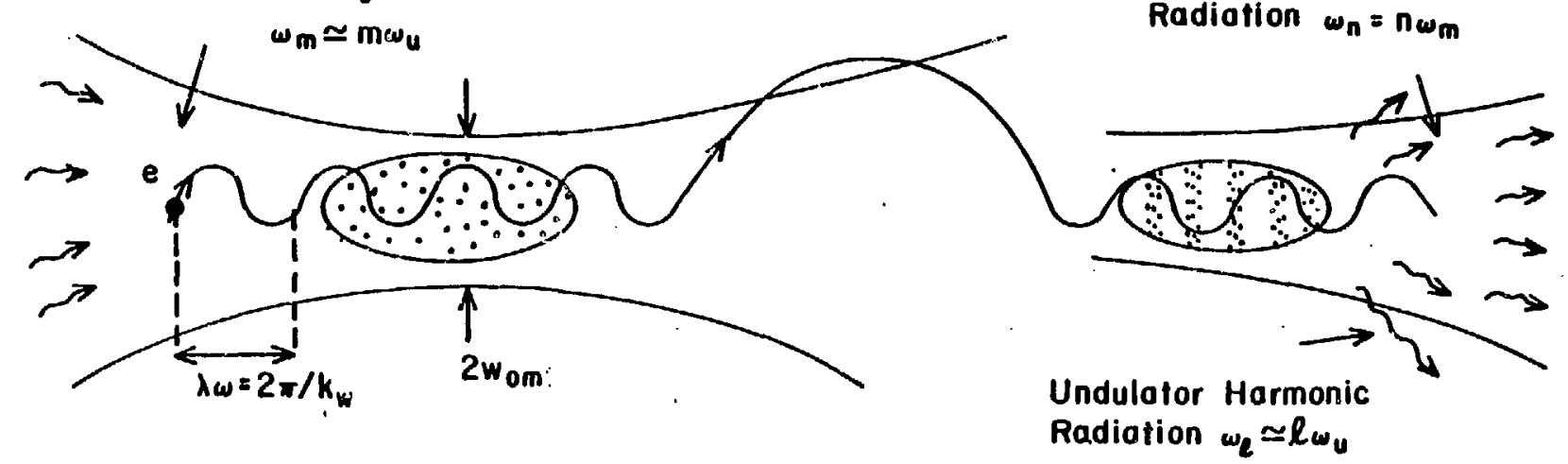

FEL CONFIGURATION

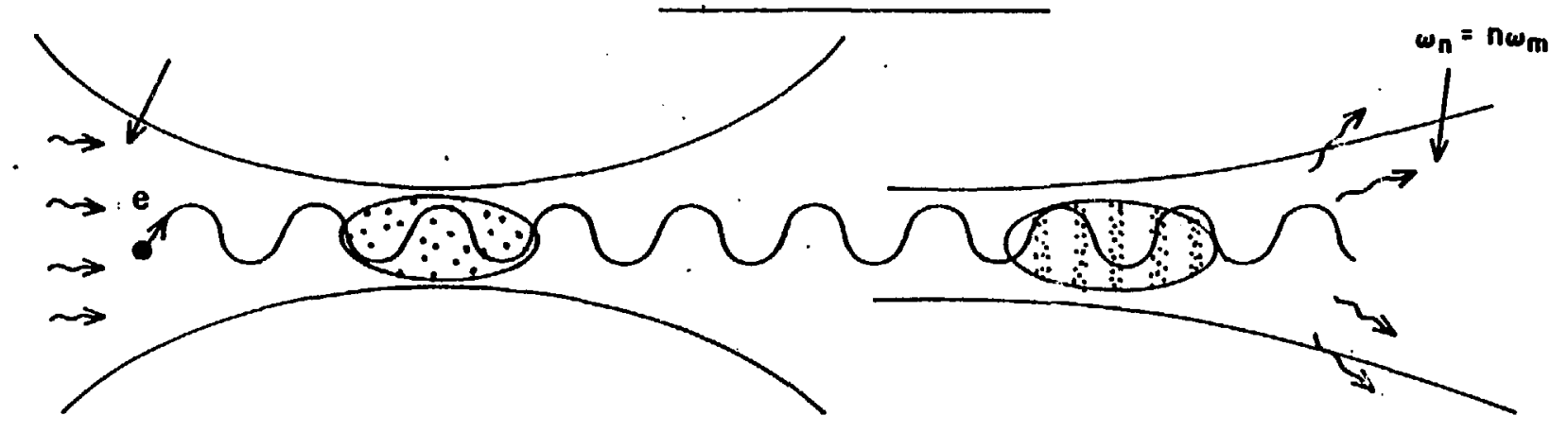


$\omega_{u}$. Since super-radiant radiation can take place only at frequencies in which the electrons emit spontaneously, both $\mathrm{n}$ and m must be odd as well. Consequently if $m \neq 1$ not all the undulator radiation frequencies can be excited super-radiantly. For example, for $m=3$ undulator harmonics $l=5,7,11,13,17, \ldots$ will not radiate super-radiantly (coherently). As is evident from the Table, the super-radiant radiation is spectrally different from the incoherent harmonic undulator radiation also in its much narrower linewidth (temporal coherence). It can thus be distinguished from the undulator radiation background by means of a high resolution spectrometer.

Fig. 1b illustrates an alternative configuration for super-radiant harmonic emission, as chosen in the NSLS experiment. It is based on the realization that the modulation, density bunching and radiation do not necessarily have to take place in separate sections. The basic idea here is that with nowadays technology even a simple uniform wiggler can be produced with strong enough magnetic field, so that not only efficient energy modulation and radiation will take place in it, but it would also provide sufficient dispersion effect to produce a substantial density bunching along the entire wiggler. Energy modulation, phase bunching and radiation take place all along the wiggler, however most of the energy modulation takes place in the upstream part of the wiggler where the laser beam is tightly focused and most intense, the phise bunching takes place mostly in the central part of the wiggler, after substantial energy modulation was achieved in the first part; and most of the super-radiant radiation takes place in the last downstream part of the wiggler, after substantial phase bunching was obtained. This process, is to a large extent, the same as the one which takes place in a free electron laser, except that in the FEL we are concerned only with the coherent radiation, which is emitted at the same frequency as the modulating laser beam and therefore can interfere constructively with it. In the present problem we are interested in the coherent emission at the higher harmonics of the laser beam, which of course cannot interfere with the laser beam and with each other.

\section{Definition of the Physical Model and the Mathematical - Numerical Problem}

The work, reported in the present publication, consisted of two parts. The first part involved the development of a physical model and an appropriate formulation for spread characterization of the undulator super-radiant emission problem. In the second part of this work, the physical model was reduced into a mathematical problem which can be readily and efficiently solved by numerical computation. The numerical code developed is general enough to account for the non negligible three dimensional features (of both the electron beam and the radiation fields) in the real experimental schemes depicted in Fig. 
la,b.

The physical problem, in its most general expression, essentially consists of solution of the coupled Maxwell equations:

$$
\begin{aligned}
& \nabla \times \mathbf{E}=\mu \frac{\partial \mathbf{H}}{\partial t} \\
& \nabla \times \mathbf{H}=\epsilon \frac{\partial \mathbf{E}}{\partial t}+\mathbf{J}
\end{aligned}
$$

and the classical force equations:

$$
\begin{aligned}
& \frac{d \gamma}{d t}=-\frac{e}{m c^{2}} \mathbf{v} \cdot \mathbf{E} \\
& \frac{d \mathbf{p}}{d t}=-e(\mathbf{E}+\mathbf{v} \times \mathbf{B})
\end{aligned}
$$

Here we neglect the possibility of space charge effects by ignoring the Poisson equation (this was considered elsewhere [5] within the framework of a one dimensional simplified model, and its neglect is well justified for all practical experimental parameters in the optical regime). Expressing the electromagnetic fields in terms of the vector potentials $\mathbf{B}=\nabla \times \mathbf{A} \quad ; \quad \mathbf{E}=-\frac{\partial \mathbf{A}}{\partial t}$ results in an alternative Hamiltonian formulation a somewhat simpler set of classical force equations instead of (2):

$$
\begin{aligned}
\frac{d \gamma}{d t} & =\frac{e}{m c^{2}} \mathbf{v} \cdot \frac{\partial \mathbf{A}}{\partial t} \\
\frac{d \mathrm{p}_{c}}{d t} & =-\frac{e^{2}}{2 m \gamma} \nabla \mathbf{A}^{2}
\end{aligned}
$$

Here $\mathbf{p}_{c}=\mathbf{p}-e \mathbf{A}$ is the electron canonical momentum, and $\mathbf{A}=\mathbf{A}_{\boldsymbol{w}}+\mathbf{A}_{\boldsymbol{m}}+\mathbf{A}_{\mathbf{s}}$ is the total vector potential field, composed of the wiggler, modulating laser beam and generated radiation field respectively.

For practical experimental parameters in the optical regime the detailed 3-D solution of the coupled sets of equations can be substantialiy simplified by two model assumptions: (1) The electron trajectories are not effected by the emitted radiation field.

(2) The electron transverse coordinates (trajectories) are only effected by the wiggler field.

The first assumption excludes the analysis from the high gain super-radiant emission regime. This regime, considered in a $1-D$ simplified model in [5], is impractical in most relevant super-radiant emission experiments in the optical regime. The second assumption is also well justified. Of course the transverse coordinates trajectories are unaffected by the relatively weak enitted radiation field, which was stated already in the first assumption to be too weak to have any effect over the electrons which generated it. But also the modulating laser beam field does not affect the transverse trajectories of the electrons; the transverse field of the modulating wave only produces very high frequency small amplitude 
transverse quiver, and the longitudinal ponderomotive force, which is the result of the beating of the laser field with the wiggler field modulates the axial velocity of the electron and affects only its axial coordinate (phase bunching).

Due to the decoupling of the electron modulation problems (Eq. 2 or 3 ) from the electron radiation problem (Eq. 1), made possible by assumption (1), one can separate the physical problem of super radiant undulator radiation into two parts: the modulation problem and the radiation problem. These are solved separately in Chapters 2 and 3 respectively.

Based on the mathematical formulation of the physical problem on its two parts, a computer numerical code was written which solves the force equations to find the trajectories of electrons which enter the interaction region at arbitrary initial conditions. The code consequently computes the required optical parameters of the electrons radiation field by performing an appropriate statistical averaging over the initial conditions distribution of the electron beam. The 3-D features of the code, listed in Table 3 , are quite extensive in both the electron beam and radiation field parameters. The model allows arbitrary dimensions, angular spread and energy spread of the electron beam. Describing the various electron distributions in terms of Gaussian functions (which is a particularly good approximation for storage ring beams), the model allows separate focusing in the two transverse $(\mathrm{x}, \mathrm{y})$ dimensions with different beam waist spot sizes $\sigma_{x}, \sigma_{y}$, and different waist positions $z_{w_{x}}, z_{w_{y}}$. Also the modulating laser beam (taken to be a fundamental Hermit Gaussian mode) can have arbitrary $x$ and $y$ focusing parameters (which can be experimentally realized with cylindrical optical components). The characterization of the emitted radiation was formulated both in terms of free space discrete modes (Hermit Gaussian set) and plane waves. Both spectral (partial temporal coherence) and phasor (full temporal coherence) formulations were used. In the computations presented in this report we used the formulation and notation of phasors and plane wave expansion.

Though the physical model is more general, we presently applied the the numerical solution of the electron trajectories to the uniform wiggler structure of Fig.1b. Straightforward extension is possible to include arbitrary magnet field small variation introduced by design or due to manufacturing errors. It can also be similarly extended to describe more complex magnetic field structures like in Fig. 1a.

\section{THE ELECTRON TRAJECTORIES PROBLEM.}

It follows from the introductory discussion that the electron transverse trajectories are simply the trajectories of an electron undulating and freely propagating in a uniform wiggler in the absence of any radiation fields. The transverse coordinates $x_{j}(t)=\bar{x}_{j}(t)+$ 
$x_{w j}(t), \quad y_{j}(t)=\bar{y}_{j}(t)+y_{w j}(t)$, are composed in general of an averaged (over undulation periods) motion, and an undulation motion $\left(x_{w j}, y_{w j}\right)$. In a planar wiggler $y_{w j}=0$, and:

$$
\begin{aligned}
& \bar{x}_{j}=x_{j 0}+c \beta_{j 0} \phi_{x_{j 0}}\left(t-t_{j 0}\right) \\
& x_{w j}=-\hat{e}_{x} \frac{a_{w}}{\gamma k_{w}} \sin \left(k_{w} z_{j}(t)\right) \\
& \bar{y}_{j}=y_{j 0}+c \beta_{j 0} \phi_{y_{j 0}}\left(t-t_{j 0}\right) \\
& \beta_{w j}=\widehat{e}_{x} \frac{a_{w}}{\gamma} \cos \left(k_{w} z_{j}(t)\right)
\end{aligned}
$$

The transverse average motion is very important in our 3-D modelling. It is responsible not only for spread in the longitudinal emission spectrum (as in the 1-D model), but also due to the transverse finiteness of the modulating laser beam, the average transverse motion may effect substantially the energy modulation (bunching) force experienced by the electron. In (4) we took for the average transverse trajectory simple straight line propagation, determined by the initial spatial and angular displacements of the electrons off the beam axis $\left(x_{j 0}, \phi_{x_{j 0}} ; y_{j 0}, \phi_{y_{j 0}}\right)$. Thi ie assumption is correct for a transversely uniform wiggler, which is a good approximation for a practical storage ring based super-radiant emission experiment with a short wiggler. Extension to a wiggler with non-negligible transverse gradient of the magnetic field is straightforward by simply replacing the straight line average trajectory (4.c) by the appropriate sinusoidal betatron trajectory in the $y$ dimension [6].

The modulating laser beam field is taken to be a general fundamental Hermit Gaussian mode with independent $x$ and $y$ focusing parameters:

$$
\begin{aligned}
\mathbf{E}_{m}(x, y, z, t)= & \widehat{e}_{x} E_{0} \frac{w_{x m}(0) w_{y m}(0)}{w_{x m}(z) w_{y m}(z)} \times \\
& \times \operatorname{Re}\left[\exp \left\{-\frac{x^{2}}{w_{x m}^{2}(z)}-\frac{y^{2}}{w_{y m}^{2}(z)}+i \Phi(x, y, z)+i \frac{\omega_{m}}{c} z-i w_{m} t\right\}\right] \\
\Phi\left(x, y, z, \omega_{m}\right)= & \frac{1}{4} \frac{\omega_{m}}{c}\left(\frac{x^{2}}{R_{x m}(z)}+\frac{y^{2}}{R_{y m}(z)}\right)-\frac{1}{2}\left[\arctan \left(\frac{z-z_{w_{x}}}{z_{R_{x m}}}\right)+\arctan \left(\frac{z-z_{w_{y}}}{z_{R_{y m}}}\right)\right] \\
R_{x, y}= & \left(z-z_{w_{x, y}}\right)\left(1+\frac{z_{R_{x, y}}^{2}}{\left(z-z_{w_{x, y}}\right)^{2}}\right) \\
{\left[z_{R}\left(\omega_{m}\right)\right]_{x, y}=} & \pi \frac{\left[w_{0 m}\right]_{x, y}^{2}}{\lambda_{m}}
\end{aligned}
$$

Note that taking this 3-D model field, already affects even electrons on axis, which now experience an electromagnetic wave field with varying amplitude and phase along the interaction length. Certainly electrons propagating off axis experience a modulating field much different from the 1-D case. 


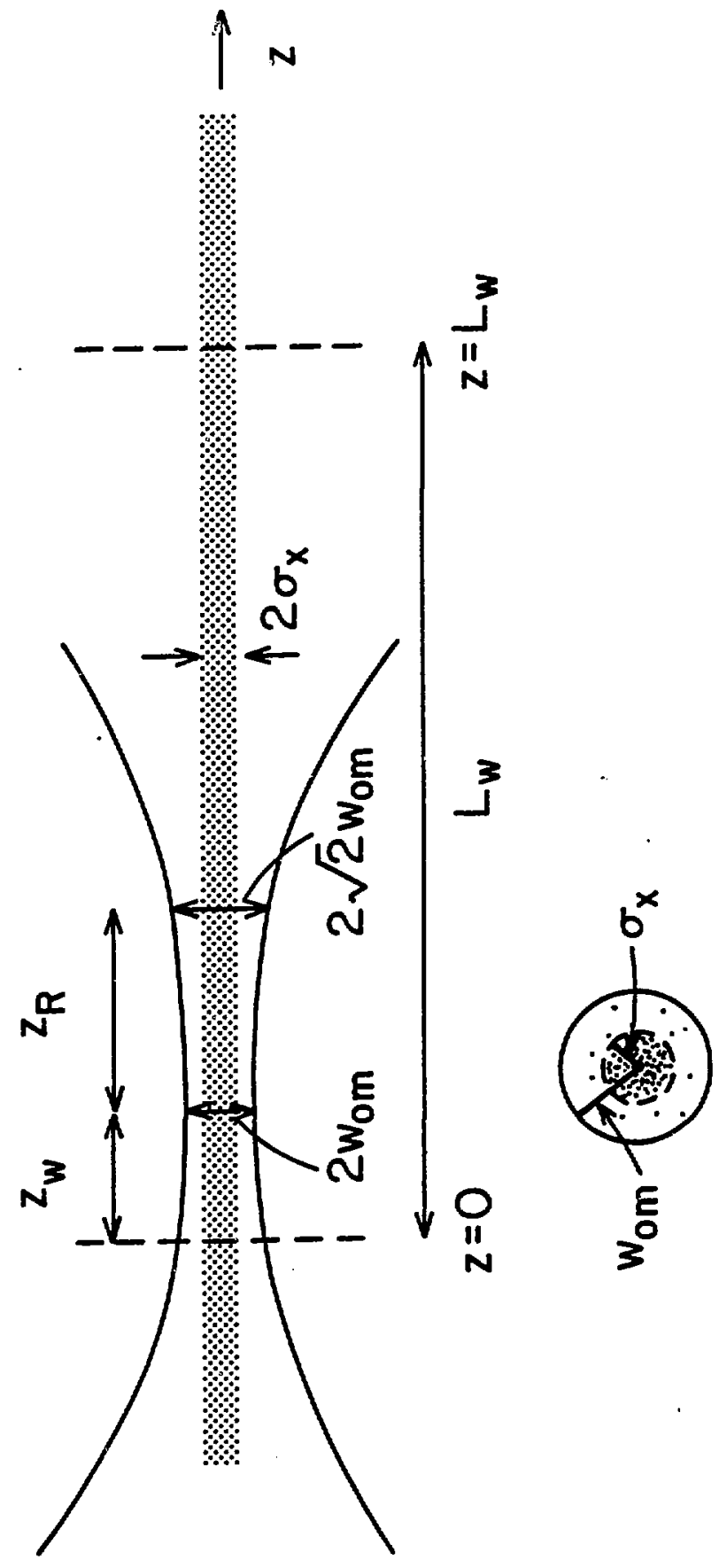

Fig. 2 Axial and transverse cross-sectional schematics of the electron beam and the modulating radiation beam. 
The longitudinal trajectory of the electron $z_{j}(t)$ is determined by the combined effect of the modulating laser beam and wiggler fields. We have found that by expressing the force equations (3) in terms of the relativistic proper time $\tau$, it can be reduced exactly (without a $\gamma \gg 1$ approximation) into a pendulum equation for the electron phase [7]:

$$
\begin{aligned}
& \left\{\begin{array}{l}
\frac{d \gamma_{j}}{d r}=\frac{1}{2}(-)^{\frac{n-1}{2}} \frac{e E_{m}\left(x_{j}(t), y_{j}(t)\right)}{m c} a_{w}\left[J_{\frac{n-1}{2}}(u)-J_{\frac{n+1}{2}}(u)\right] \sin \left[\psi_{m j}+\Phi_{m j}\left(x_{j} y_{j} z_{j} \omega_{m}\right)\right] \\
\frac{d \psi_{m j}}{d r}=\frac{k_{m}+m k_{w}}{m} p_{z}-\omega_{m} \gamma \\
{\left[J_{\frac{n-1}{2}}(u)-J_{\frac{n+1}{2}}(u)\right] \omega_{m} p_{z}-\left[\left(k_{m}+k_{w}\right) J_{\frac{n-1}{2}}(u)-\left(k_{m}-k_{w}\right) J_{\frac{n+1}{2}}(u)\right] m c^{2} \gamma=\text { const }_{j}}
\end{array}\right. \\
& \psi_{m j} \equiv\left(k_{m}+m k_{w}\right) z_{j}(t)-\omega t \\
& \tau \equiv \int_{t_{j 0}}^{t} \frac{d t^{\prime}}{\gamma_{j}\left(t^{\prime}\right)}=\text { proper time } \\
& u \equiv \frac{\omega_{m}}{8 k_{w}} \frac{a_{w}^{2}}{c \beta_{z} \gamma^{2}} \approx \frac{m}{4} \frac{a_{w}^{2}}{1+a_{w}^{2} / 2}
\end{aligned}
$$

This set of equations needs to be solved with the initial conditions: $\psi_{j}(\tau=0)=$ $\psi_{j 0}=-\omega t_{j 0} ; \quad \gamma_{j}(\tau=0)=\gamma_{j 0} ; \quad z_{j}(\tau=0)=0 \quad$ It is basically a pendulum equation with slowly varying amplitude and phase, which depend on the electron transverse trajectories. The fast longitudinal quiver (which is the essential source of the odd harmonic undulator radiation emission when $K \ll 1$ ) was averaged away in the derivation of Eq. 6 (off the force equations), leaving the various Bessel function factors, and producing the average constant of the motion of Eq. (6c) [8].

A fast integration numerical solution code called PENTOK was written to solve the differential equations (6) using the Gear integration method [9]. The program was run on the B.N.L. CDC 7600 computer and we show in Figs 3,4 some exemplary computation results based on the parameters of Table 4. The data of the table relates to a uniform wiggler structure as shown in Fig. 1a. The schematic iaser beam and electron beam focusing parameters are illustrated in Fig. 2. For the sake of simplicity zero spread parameters were assumed in this first example. The electron beam propagates on axis with diminishing narrow width and no energy spread. The laser beam (at the frequency of a doubled Nd-YAG laser) is illuminating the electrons in the wiggler at the third $(\mathrm{m}=3)$ harmonic of the fundamental undulator frequency. It is assumed to be circularly symmetric. and focussed at the up-stream part of the wiggler at $z_{w}=30 \mathrm{~cm}$.

Fig. 3 displays the positions in energy-phase space of 36 electrons, initially equi-spaced in phase, shown at four points along the wiggler: $z=0,0.5,1$ and $2.2 \mathrm{~m}$. The electron beam energy tune for this run $(\gamma=785.64)$ corresponds to maximum bunching at the end of the wiggler whicin was found to be $0.32 \%$ higher than the 1-D synchronization energy $\left(\gamma_{R}=\left(1+\bar{a}_{w}^{2}\right) \lambda_{w} /\left(2 m \lambda_{m}\right)=783.14\right)$. This 3-D effect is explained in sect. 4 . In the first 


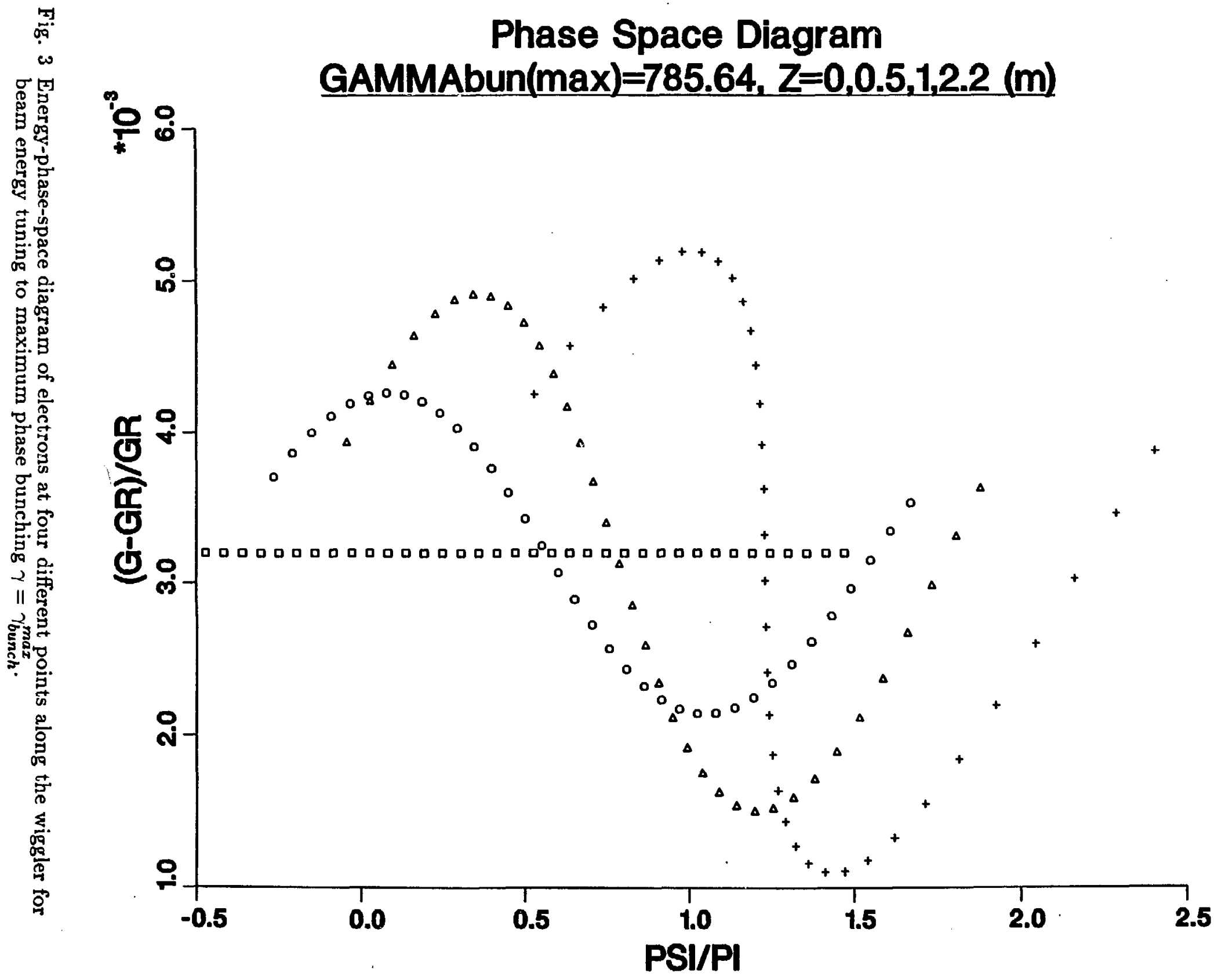




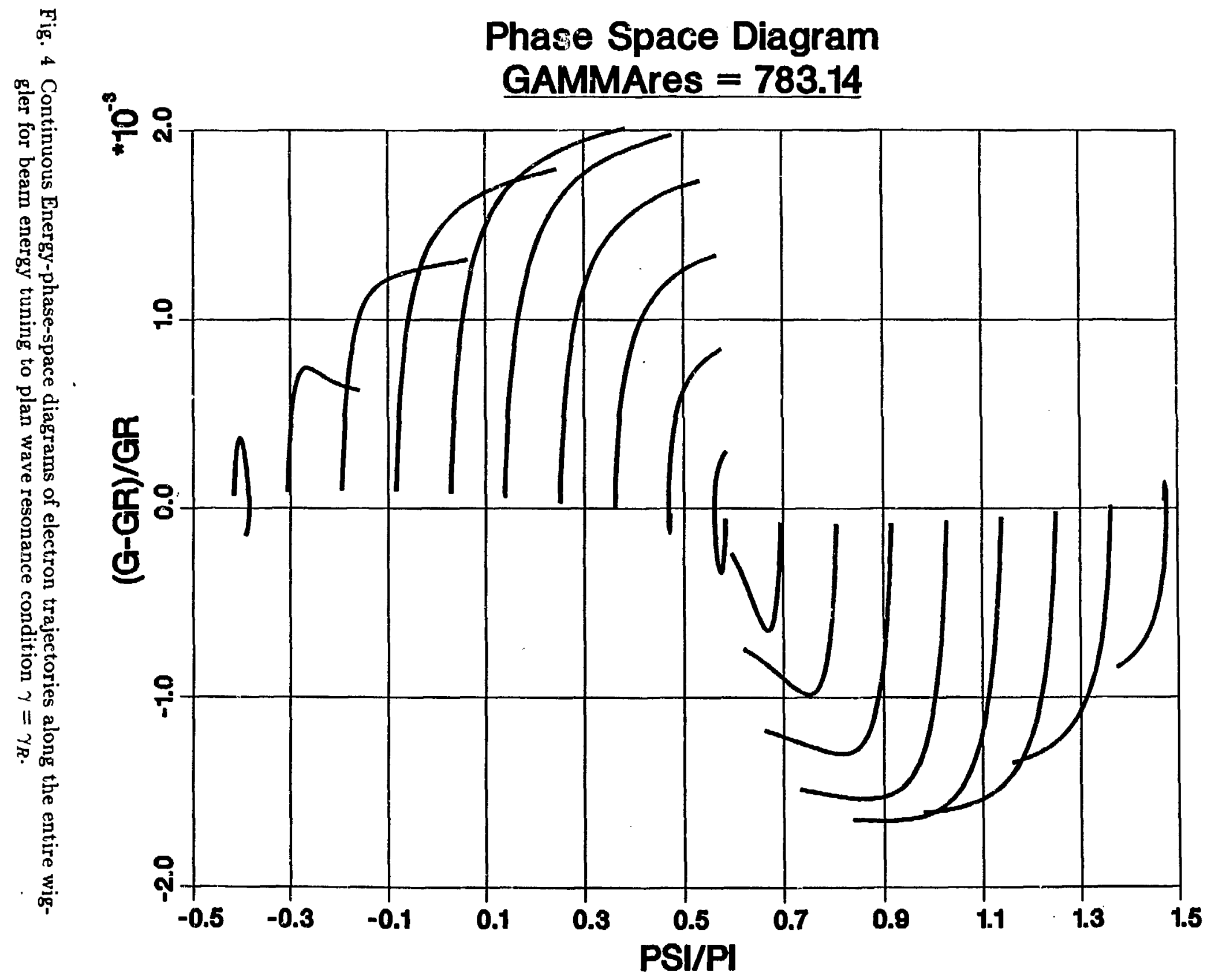


part of the wiggler ( $u p$ to $z=1 \mathrm{~m}$ ) the electrons are modulated mostly in energy, and their phases do not change much. Only in the last part of the wiggler phase bunching, induced by the dispersive propagation along the wiggler, becomes significant. We also observe that in the last part of the wiggler most of the electrons experience a positive phase drift which is a result of operating at $\gamma>\gamma_{R}$

The synchrotron oscillator period of the electron inside the pendulum potential well (the wiggler length at which a well trapped electron would complete a full revolution in phase space) was found to be $z_{s y n c}=7.1 \mathrm{~m}$, based on the optimistic assumption that a uniform laser beam field equal to the maximum intensity in the waist point can be kept along the entire wiggler length. Though the laser beam Rayleigh range (1m) is shorter than $\lambda_{\text {synch }} / 4$, substantial phase bunching was obtained because of the highly dispersive propagation along the wiggler in the low laser field regions at the end of the wiggler We observe in Figs. 3 that in the present example an almost maximal phase bunching takes place at the end of the wiggler, but the maximum bunching point was not yet reached. Evidently saturation in the super-radiant harmonic emission level would require a slightly longer wiggler.

Fig. 4 illustrates in a continuous line presentation the phase space trajectories of 18 electrons at beam energy $\gamma=\gamma_{R}$. Clearly less bunching effect takes place at this energy. The continuous line phase space curves presentation of Fig.4 also reveal a curious behavior. The electrons near the center of the pendulum potential well ("bucket") first decelerate and after some distance along the wiggler they accelerate. The same kind of movement, but in the reverse order is exhibited by the electrons in the bucket borders $(\psi=-.5 \pi, 1.5 \pi)$. The center bucket electron trajectories might have been considered erroneously to be a section of slightly more than half a period of synchrotron oscillation phase-space revolution. Such an hypothesis would be incorrect since the wiggler length $(2.2 \mathrm{~m})$ is shorter than the lower bound estimate of half the synchrotron period $(3.55 \mathrm{~m})$, and it would not explain the behavior of the electron near the bucket borders. This curious behavior is a real 3-D effect which we termed "the Gaussian phase shift jolt effect". It is explained in terms of a simple mechanical pendulum analogy in Fig. 5.

The Gaussian phase jolt effect is related to the phase shift $\Phi$ (Eq. 5) of the Gaussian mode of the modulating laser beam. An electron going on axis, starting from a point at which the laser beam still converges towards its waist, will experience first a small negative temporal phase shift in the yendulum equation force (6a) (as compared to the constant phase ponderomotive force produced by an ideal harmonic plane wave whose phase front coincides with the Gaussian phase front at the waist point, and shifts by $\pm \pi / 2$ at $\pm \infty$ ). This phase shift turns to be positive after the waist position. The maximum phase shift 

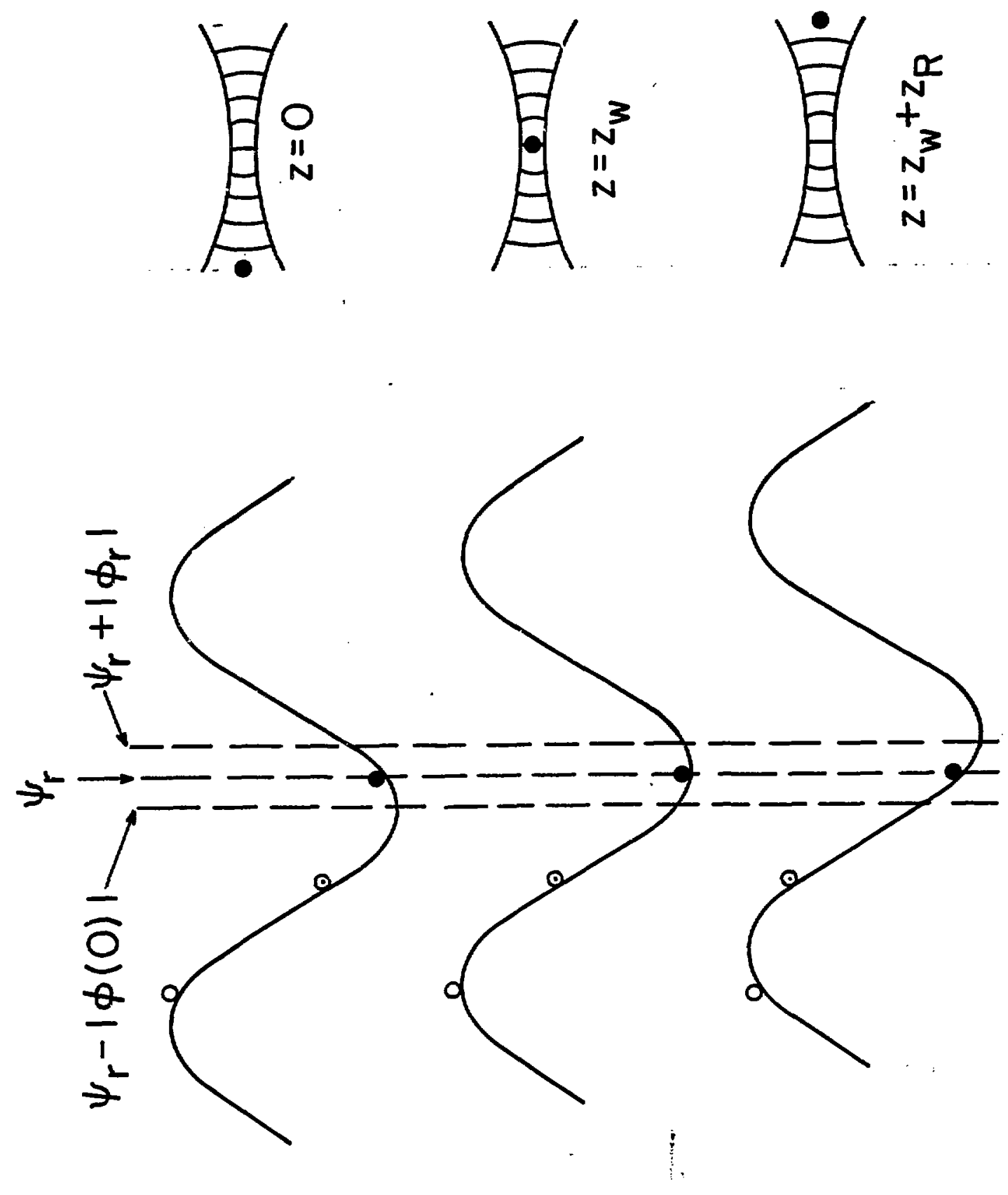

Fig. 5 Mechanical analogue diagram of the Gaussian phase "jolt effect". Only the highly stable and unstable particles experience reversal of force direction throughout the process. 
that would take place as the electron moves from- $\infty$ to $\infty$ is $\pi$. This is a very slow phase change, however half of it occurs in the section $-z_{R}<z<z_{R}$ which is smaller in our case relative to the synchrotron (pendulum) oscillation period! In the pendulum equation time scale the slow Gaussian phase shift looks like a very fast movement of the center of oscillation of the pendulum force ("jolt"). This behavior is depicted in the mechanical analogue picture of Fig. 5 by three "test particles". The drawing clearly illustrates why predominantly the stable point and unstable point particles are most susceptible to the jolt effect, which reverses the direction of the force applied on them as they go through the zero phase shift (Gaussian beam waist) point. The other particles experience only a small relative change in the force applied on them, which at no place reverses the direction of the force they experience and the direction of their motion. Though somewhat of a mathematical curiosity, we suspect that this 3-D effect may have also practical implication in effecting the efficiency of high order super radiant harmonic emission, which is strongly dependent on the electron density near the phases of the center and ends of the bucket.

\section{THE ELECTRON RADIATION PROBLEM}

The study of the dynamics of the electrons in the 3-D fields of the wiggler and the laser beam produced interesting and expectedly useful results, but our main thrust in this work is to study the radiation problem. For analyzing the radiation problem there are two kinds of formulations that one can use. One approach is a spectral formulation in which the fields and currents are decomposed in frequency domain by a Fourier transform:

$$
\begin{aligned}
& \check{\mathbf{E}}(\mathbf{r}, \omega)=\int_{-\infty}^{\infty} \mathbf{E}(\mathbf{r}, t) e^{\imath \omega t} d t \\
& \check{\mathbf{J}}(\mathbf{r}, \omega)=\int_{-\infty}^{\infty} \mathbf{J}(\mathbf{r}, t) e^{i \omega t} d t
\end{aligned}
$$

The other approach - the phasor formulation - is based on decomposition of the fields and currents into a Fourier series, which consists of a DC term and an infinite sum of discrete terms at the external force frequency and all its harmonics:

$$
\begin{gathered}
\mathbf{E}(\mathbf{r}, t)=\operatorname{Re} \sum_{n=1}^{\infty} \tilde{\mathbf{E}}\left(\mathbf{r}, n \omega_{m}\right) e^{-\imath n \omega_{m} t} \\
\mathbf{J}=J_{0}+\operatorname{Re} \sum_{n=1}^{\infty} \tilde{\mathbf{J}}_{n} e^{-i n \omega_{m} t}
\end{gathered}
$$

This formulation is rigorously applicable to any nonlinear response problem which is independent of time (temporal translation symmetric), when a single frequency harmonic force 
is applied to the system. It is a good approximation for the present problem when finite pulse effects can be ignored, so that the laser beam can be considered a continuous harmonic coherent wave, and the electrons can be modelled by an infinite continuous beam; and furthermore, the electrons shot noise (current fluctuation due to the corpuscular nature and random spatial distribution of the electron beam) is ignored.

With either of these formulations we have yet to suggest a formulary bridge between the microscopic (single electron) approach used in the previous chapter to characterize the electron propagation, and the macroscopic (fluid current or current field) definition used in the Maxwell equations (1) and in Eqs. (8),(10).

We have developed the spectral approach in some detail in a previous publication [7]. We consequently will only describe here the main results of this formulation, which help to understand the spectral characteristics (temporal coherence) of the super-radiant radiation. After the spectral characteristics are understood, it is preferable to use phasor the formulation, in order to describe the emitted radiation with minimal parameters, and in order to simplify the numerical computation.

To connect the single electron approach results to the field approach we first express the current field as a sum of delta functions:

$$
\mathbf{J}=\sum_{j=1}^{N(t)}-e \mathbf{v}_{j}(t) \delta\left(\mathbf{r}-\mathbf{r}_{j}(t)\right)
$$

$N(i)$ is the number of electrons in the system up to time $t$. In the spectral formulation we usually deal with signals of finite duration, and $N$ would be in the present problem the total number of electrons in a pulse. When dealing with random stationary processes, like the incoherent undulator radiation emission, $\mathrm{N}$ can be taken to be the average number of electrons entering the interaction region during a characteristic time of the random process statistics. In free electron spontaneous radiation schemes with a continuous electron beam (or long pulse), this characteristic time may be taken in single electron interaction problems (excluding cooperative interaction regimes like amplified spontaneous emission) to be the undulation period $2 \pi / \omega_{u}$. Eq. (11) can be now substituted in (8) to produce an expression for the spectral current of the beam:

$$
\check{\mathbf{J}}(\mathbf{r}, \omega)=-e \sum_{j=1}^{N} \delta\left(\mathbf{r}_{\perp}-\mathbf{r}_{\dot{\perp}}\right) \frac{\mathbf{v}_{j}}{v_{z j}} e^{i \omega t_{j}(z)}
$$

which can be used in the Fourier transformed formulas of Maxwell equations.

Even though they are now time independent, the Fourier transformed Maxwell equations with the current source term (12) are still a difficult to solve set of 3-D partial 
differential equations. The problem may be considerably simplified by expanding all the fields in terms of an orthogonal complete set of modes of the electromagnetic structure (free space in the present problem). Again we have a choice to make, whether to use a discrete set of modes (e.g. Hermit-Gaussian modes) or a continuous set (e.g. plane waves). The first choice is more favorable if we have reasons to believe that the emitted radiation can be well described by a small number of discrete radiation modes We will make this choice in the context of the present discussion on the spectral formulation results; but in the subsequent phasor formulation discussion and in the numerical computations the other choice (plane wave expansion) is taken.

Substituting in the Fourier transform of the Maxwell equations (1) the general expansion of the radiation field in terms of an infinite set of discrete orthogonal modes, one can derive explicit expressions for the expansion coefficients by taking advantage of the modes orthogonality and using the expression for the discrete particles current (11):

$$
\begin{aligned}
& \check{\mathbf{E}}(\mathbf{r}, \omega)=\sum_{q} \check{c}_{q}(\omega) \widetilde{\mathbb{E}}_{q}(\mathbf{r}) \\
& \check{c}_{q}(\omega)=-\frac{1}{P_{q}} \Delta \check{W}_{q}(\omega) \\
& \Delta \check{W}_{q}=\sum_{j=1}^{N} \Delta \check{W}_{\mathbf{q} j}(\omega) \\
& \Delta \check{W}_{\mathbf{q} j}(\omega)=-e \int_{t_{0 j}}^{t_{j}\left(L_{w}\right)} \tilde{\mathbf{E}}_{q}^{*}\left(\mathbf{r}_{j}(t)\right) e^{i \omega t} \cdot \underline{u}_{j}^{j}(t) d t
\end{aligned}
$$

where $\tilde{E}_{q}$ are the expansion modes and $P_{q}$ - their normalization powers.

This general solution of the radiation problem has a simple physical interpretation which is illustrated in Fig. 6. The amplitude of each radiation mode in the infinite sum is proportional to an infinite sum of contributions to this mode by the different electrons in the beam $-\Delta \check{W}_{\mathbf{q}} \quad(j=1, \ldots, N)$. These terms have the dimension of energy but they are complex numbers. The real part of $\Delta W_{q j}$ can be interpreted as the work done by electron $e_{j}$ on the mode $\mathrm{q}$ during the the electron transit time $t_{j}\left(L_{w}\right)-t_{n j}$. By conservation of energy, this work is equal to the energy in the longitudinal wavepacket emitted by the electron into mode q. Fig. 6 displays two wavepackets emitted by two electrons $e_{j 1}, e_{j 2}$, which enter and exit the wiggler at different times. The length of each wavepacket is the slippage distance along the wiggler $-N_{w} \lambda_{u}$ (considering now emission at the fundamental undulator harnionic frequency). In frequency domain the two wavepackets contribute additively to the total spectral field of the mode, and their contributions add constructively or destructively if the spacing between the two wavepackets $\left(c\left(t_{j 2}\left(L_{w}\right)-t_{j_{1}}\left(L_{w}\right)\right)\right)$ is correspondingly an 


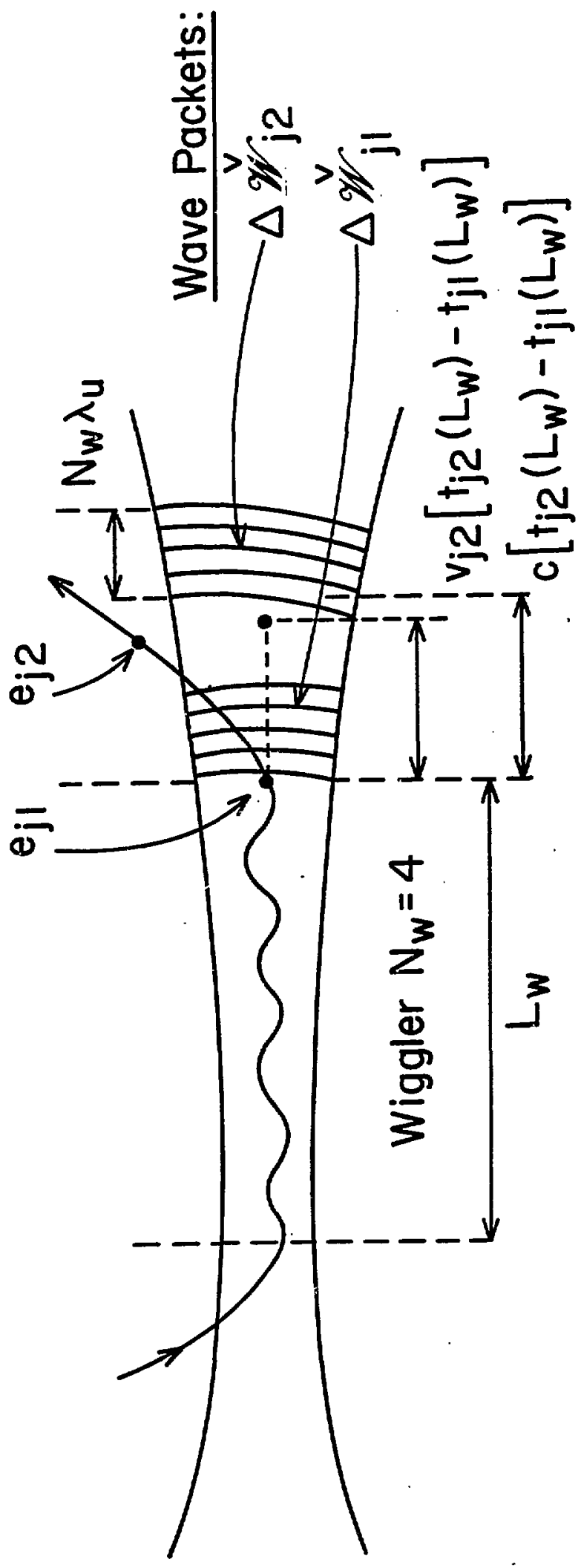

Fig. 6 Schematic illustration of wave packet emission into a transverse radiation mode by single electrons. The diagram depicts an example of a four period wiggler and corresponding four period optical wavepacket. 
even or odd integral multiple of half the wavelength of the spectral radiation component under consideration. The argument of the "complex work" parameter $\delta \dot{W}_{\mathbf{q} j}$ measures the phase of each wavepacket relative to the phase of the radiation mode, so that the algebraic sum in (13b) measures the coherent contribution of all electrons to the mode amplitude at frequency $\omega$. Note that if the phases of the different electrons stay random along the entire interaction length this, sum will result in on the average a vanishing contributions as expected. only when the electrons get bunched in phase either right at the entrance or at least within the interaction region, their spectral radiative contributions can add constructively to build a nonvanishing spectral component of the mode amplitude.

The solution (13) is in fact a complete general solution of the radiation problem, which can even give the entire time dependent radiation field at any point in space after an inverse Fourier transform is applied to (13a). The complex work elements in (13d) can be straightforwardly computed numerically by substituting in it the electrons trajectories $\mathbf{r}_{j}(t)$ and velocities $\mathbf{v}_{j}(t)$ which were computed in the first part of the interaction problem (previous chapter) and performing the time integration. However such representation of the final solution is too general, consumes immense computation time, and is difficult to display. More limited and concise representation in terms of a small number of optical parameters is necessary in order to make the the solution useful for practical implementation.

It is costumery in optics to characterize the basic optical properties of a partially coherent radiation source in terms of the spectral brightness parameter of the source and other parameters that can be derived from it, like the radiant intensity, the power and the corresponding spectral parameters. The spectral energy brightness of a pulsed source can be described in general in terms of the Wigner distribution of the fields [10]. When we assume that only a single mode is excited (which means also that the radiation is spatially coherent) the only parameter left to characterize the spectral properties of the radiation is the momentary spectral power in the mode which is expressed in terms of the Wigner function $W_{C_{q}}[7]$ :

$$
\begin{aligned}
& \frac{d P_{q}}{d \omega}=\frac{P_{q}}{\pi} W_{c_{q}}(\omega, t) \\
& W_{c_{q}}(\omega, t) \equiv \frac{1}{2 \pi} \int_{-\infty}^{\infty} d \Omega\left\langle\check{c}_{q}\left(\omega+\frac{\Omega}{2}\right) \check{c}_{q}^{*}\left(\omega-\frac{\Omega}{2}\right)\right\rangle e^{-i \Omega t}
\end{aligned}
$$

This parameter can be calculated once the spectral amplitude of the mode (13b) is computed for a range of frequencies.

The spectral brightness computation is described in some more detail in [7]. Fig.7 describes qualitatively the on axis radiation spectral brightness distribution in time frequency phase space, comparing the incoherent emission limit (Fig. 7a) to the ideal tight bunching 
Time-Frequency Phase Space Picture

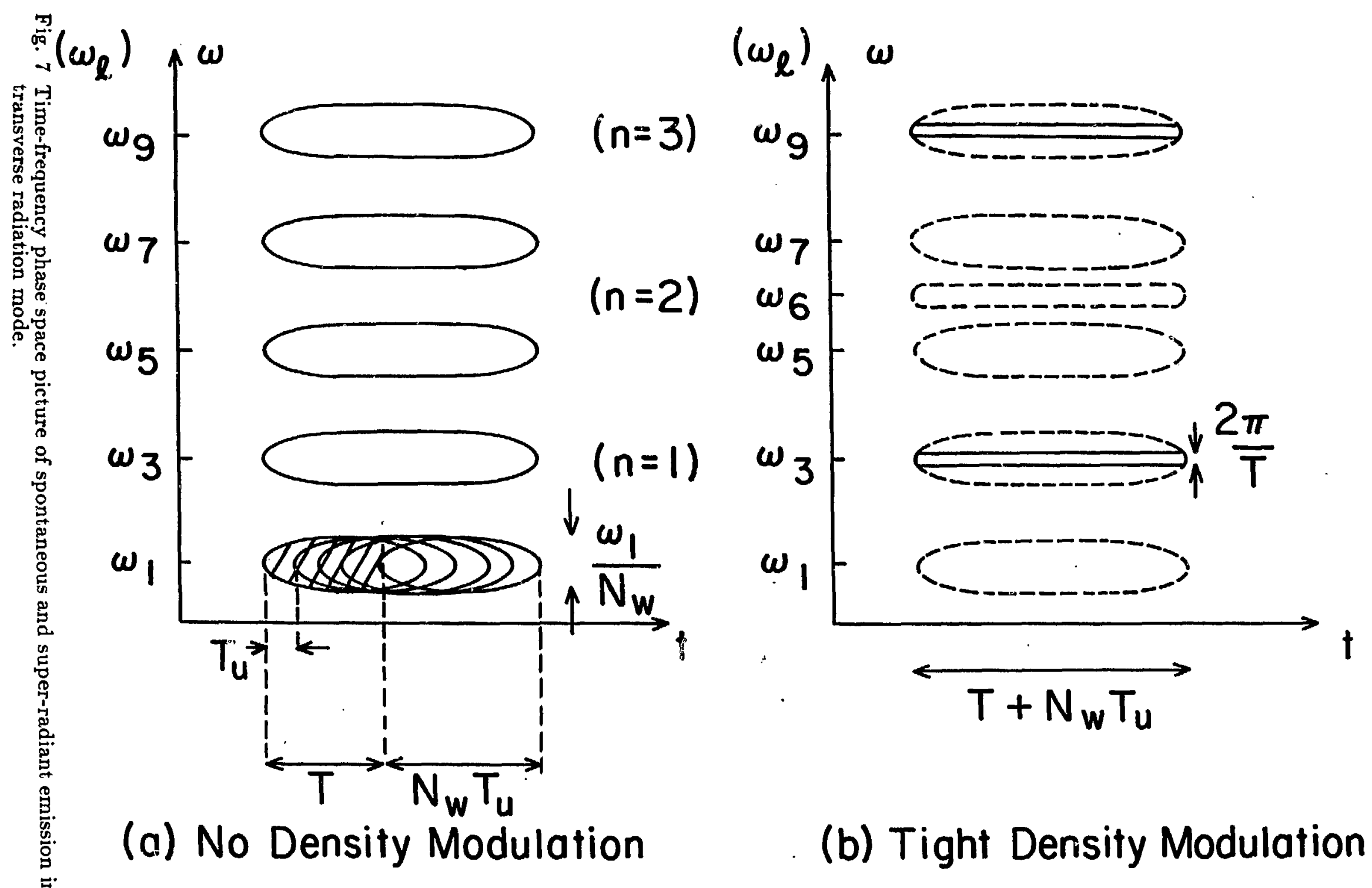


limit (Fig $7 \mathrm{~b}$ ) for the example $m=3$. The radiation pulse duration is approximately equal in both cases to the electron pulse duration $\mathrm{T}\left(\equiv T_{\text {pulse }}\right.$ ) plus the slippage time $N_{w} T_{u}$ (which is usually negligible even though in the figure it is exaggeratedly long). However the spectral width of the super-radiant radiation substantially shrinks from $\omega_{u} / N_{w}$ to $2 \pi / T$, which for the parameters of Table 4 and an electron pulse duration $T=0.5 n s e c$ corresponds to a significant reduction in time-frequency phase space area of $4.3 \times 10^{3}$. This number gives some indication on the enhancement in spectral brightness when superradiant emission is set. Note though that in practice, full bunching is never obtained and therefors there will always be some incoherent spontaneous emission measured together with the coherent super-radiant emission. On the other hand note that Fig. 7 does not describe the other four dimensions of phase space, and that in the ideal bunching case the spectral brightness improvement factor is even larger (by a factor $m N_{w}$ ), because there is no emission then at any angle at frequencies different than $n \omega_{m}$ The lower frequency emission at higher angles within the relativistic radiation cone (of angle $1 / \gamma_{z}$ ) disappears due to destructive interference, and all the emitted photons can be only measured in the forward direction (within a diffraction limited radiation cone). The disappearance of the $l=1,5,7, \ldots$ harmonics in the on axis spectrum is depicted in Fig. $7 \mathrm{~b}$ by the broken line regions.

The spectral formulation is helpful for a simultaneous description of the (temporally) incoherent and coherent radiation and for studying the transition from one limit to the other, when the emission process becomes more and more super-radiant as the bunching is enhanced. However if we are predominantly interested in the coherent radiation, and after understanding already that it is emitted in a very narrow bandwidth around the laser harmonic frequencies, and that its coherence length is Fourier transform limited by the finite duration of the electron beam pulse, it is more convenient to switch over to a phasor formulation, neglecting the finite pulse effects, and ignoring the shot noise. In the phasor formulation we decompose the fields and currents into an infinite sum of terms oscillating at the modulating laser frequency $\omega_{m}$ and its harmonics $(9,10)$. For a discrete charged particles current (electron beam) the current phasor components. obtained from periodic Fourier series decomposition of (11) are:

$$
\begin{aligned}
& \mathbf{J}_{0}=-\frac{e}{T_{m}} \sum_{j=1}^{N_{T_{m}}} \delta\left(\mathbf{r}_{-}-\mathbf{r}_{-j}\right) \frac{\mathbf{v}_{j}}{v_{z j}} \\
& \tilde{\mathbf{J}}_{n}=-\frac{2 e}{T_{m}} \sum_{j=1}^{N_{T_{m}}} \delta\left(\mathbf{r}_{-}-\mathbf{r}_{-j}\right) \frac{\mathbf{v}_{j}}{v_{z j}} e^{2 n \omega_{m} t_{j}(z)}
\end{aligned}
$$

The use of the electron trajectories $\mathbf{r}_{j}(t), \mathbf{v}_{j}(t)$ computed before in the single electron 
formalism of the previous chapter (including the inverted longitudinal coordinate trajectory function $t=t_{j}(z)$ ), makes it possible to have an explicit formal expression of the phasors current fields (15). In these expressions $N\left(T_{m}\right)$ is the average number of electrons entering the interaction length in one optical period of the laser (shot noise fluctuations are ignored). The current phasors (15) together with the set of time independent partial differential equations obtained by substitution of the fields' phasor decomposition (9) in the Maxwell equations (1), form now a well defined mathematical problem of 3-D partial differential equations with a source term.

To proceed on in solving the set of partial differential equations we can expand all the fields in terins of either a discrete or a continuous set of orthogonal modes of free space (as in the spectral formulation). In fact the spectral formulation solution can be directly transformed into a phasor formulation notation by applying the formal substitution -

$$
\rightarrow \sim \quad \sum_{j=1}^{N} \rightarrow \frac{1}{T_{m}} \sum_{j=1}^{N_{T_{m}}} ; \quad \omega \rightarrow n \omega_{m}
$$

Since we prefer to use in the phasor formulation a continuous mode (plane wave) expansion. we will not use presently the transformed discrete modes decomposition (13). The expansion of the radiation field in terms of plane waves (written already in the phasor notation) and the solution for the continuous expansion coefficients are given by

$$
\begin{aligned}
& \tilde{\mathbf{E}}=\frac{1}{(2 \pi)^{2}} \int_{-\infty}^{\infty} d^{2} q_{\perp} \sum_{\sigma} \widetilde{c}_{\sigma}\left(\mathbf{q}_{\perp}, z, n \omega_{m}\right) \varepsilon_{0} \hat{e}_{\sigma} e^{i \mathbf{q} \cdot \mathbf{r}} \\
& \tilde{c}_{\sigma}\left(\mathbf{q}_{-}, z, n \omega_{m}\right)=-\frac{1}{4 S_{0} \hat{e}_{q} \cdot \hat{e}_{z}} \Delta \widetilde{W}_{\mathbf{q}}\left(n \omega_{m}\right) \\
& \Delta \widetilde{W}_{\mathbf{q}}\left(n \omega_{m}\right)=\frac{1}{T_{m}} \sum_{j=1}^{N_{T_{m}}} \Delta \widetilde{W}_{j \mathbf{q}}\left(n \omega_{m}\right) \\
& \Delta \widetilde{W}_{j \mathbf{q}}\left(n \omega_{m}\right)=-e \int_{t_{0 j}}^{t_{j}(L)} \varepsilon_{0} e^{i n \omega_{m} t-\imath \mathbf{q} \cdot \mathbf{r}_{j}(t)} \hat{e}_{\sigma}^{*} \cdot \mathbf{v}_{j}(t) d t
\end{aligned}
$$

where $S_{0}$ is the Poynting vector power density normalization of the mode, and the summation over $\sigma$ is a summation over two orthogonal polarization states. This solution is completely analogous to the discrete mode expansion solution (13).

Using a Wigner distribution in the space dimensions, (16) can be used to compute the brightness of the radiation field from which all other radiation parameters can be calculated [11]. This would require however evaluation of $c_{\mathbf{q}_{-}}$over a range of $\mathbf{q}_{+}$values involving exhaustive numerical computation. At present we limit our optical characterization of the emitted radiation to the computation of the less general optical parameter of radiant 
intensity. Anyway, this parameter is the one which is usually measured in the synchrotron radiation lab (with a single pin-hole measurement in the far radiation field region), and would be most appropriate for comparing to the background incoherent undulator radiation which is emitted in the experiment simultaneously with the coherent radiation. The radiant intensity. is expressed directly in terms of the modulus of the plane wave amplitude by

$$
\frac{d P}{d \Omega}=\frac{\cos \Theta}{\left(\lambda_{m} / n\right)^{2}} \frac{1}{2} \sqrt{\frac{\epsilon_{0}}{\mu_{0}}}\left|\tilde{c}_{\sigma}\left(\mathbf{u}_{\perp}\right)\right|^{2}
$$

where $\left|q_{\perp}\right|=(\omega / c) \sin \Theta$ and $\Theta$ is the emission direction.

The continuous expansion coefficients $c_{\sigma}(q .).(16 \mathrm{~b})$ can be rewritten in a more explicit form in terms of the current phasor $(15 \mathrm{~b})$ :

$$
\begin{aligned}
c_{\sigma}^{\left(n \omega_{m}\right)}\left(\mathbf{q}_{\perp}, z\right) & =\frac{1}{4 \hat{e}_{q} \cdot \hat{e}_{z}} \frac{1}{S_{0}} \iiint d^{3} r \tilde{\mathbf{J}} \cdot \hat{e}_{\sigma}^{*} \varepsilon_{0} e^{-i \mathbf{q} \cdot \mathbf{r}}= \\
& =\frac{1}{4 \hat{e}_{q} \hat{e}_{z}} \frac{\varepsilon_{0}}{S_{0}} \int_{0}^{L_{w}} d z e^{-i q_{z} z} \iint d x d y e^{-i \mathbf{q} \cdot \mathbf{r}_{\perp}} \tilde{\mathbf{J}}^{\left(n \omega_{m}\right)} \cdot \hat{\varepsilon}_{\sigma}^{*}
\end{aligned}
$$

In the special case of emission on axis $\left(q_{\perp}=0\right)$ this can be written in the form

$$
\begin{aligned}
& c_{\sigma}(z)=\frac{\epsilon_{0}}{S_{0}} \int e^{-n \frac{\omega_{m}}{c} z} \widetilde{\mathbf{I}}_{\perp}^{\left(n \omega_{m}\right)}(z) \cdot \hat{e}_{\sigma}^{*} d z \\
& \tilde{\mathbf{I}}_{\perp}(z) \equiv \iint d x d y \tilde{\mathbf{J}}_{\perp}(x, y, z)
\end{aligned}
$$

where $\tilde{\mathbf{I}}_{\perp}$, having dimensions of a current, is a vector parameter which can be interpreted as a continuous polarization vector like density.

Substituting the discrete particles current phasor expression (15b) in (19), using the expression of the wiggler quiver velocity (4d) for the transverse velocity of the particle, and averaging over the fast longitudinal quiver motion (which produces another Bessel function factor), the excitation current $\tilde{I}_{\perp}$ finally can be expressed in terms of a bunching parameter $\eta_{n}$ which is simply the Fourier series component of the longitudinal current at harmonic frequency n normalized to the DC-current:

$$
\begin{aligned}
& \widetilde{\mathbf{I}}_{\perp}^{\left(n \omega_{m}\right)}=\frac{1}{2} I_{0} \frac{\mathbf{v}_{w}}{v_{z 0}}[J J]_{\ell} e^{i n \frac{\omega m}{c} z} \eta_{n} \\
& \eta_{n} \equiv \frac{\tilde{I}_{z n}}{I_{0}}=2\left\langle e^{-i n \psi_{m j}}\right\rangle_{j}
\end{aligned}
$$

where $[J J]_{l} \equiv J_{\frac{l-1}{2}}\left(l u_{0}\right)-J_{\frac{l+1}{2}}\left(l u_{0}\right), u_{0} \equiv \frac{1}{2} \frac{\bar{a}_{w}^{2}}{1+\bar{a}_{w}^{2}}$, and $\psi_{m j}$ is defined in (6d). The $\psi_{m j}$ are the results of the numerical solution of the pendulum equation in the first part of this work. The \langle\rangle$_{j}$ indicates statistical averaging over the initial conditions of the electrons in the beam. 
The amplitude of the radiation field on axis can now be expressed in terms of an integral over the density parameter along the wiggler:

$$
c_{\sigma}^{\left(n \omega_{m}\right)}(z)=\frac{1}{8} \frac{\varepsilon_{0}}{S_{0}} l_{0} \frac{v_{w}}{v_{z 0}}(-)^{\frac{l-1}{2}}[J J]_{l} \int_{0}^{z} \eta_{n}(z) \dot{d} z
$$

Substituting $:$ (17) we obtain an explicit expression for the radiant intensity expressed in terms of a normalized radiation parameter $r_{n}$ :

$$
\begin{aligned}
& \left(\frac{d P_{S . R .}}{d \Omega}\right)_{\Theta=0}=\frac{1}{16} \sqrt{\frac{\mu_{0}}{\epsilon_{0}}}\left(n \frac{\lambda_{w}}{\lambda_{m}}\right)^{2} I_{0}^{2}\left(\frac{\bar{a}_{w}}{\gamma}\right)^{2}[J J]_{l}^{2} r_{n}(z) \\
& r_{n}(z) \equiv\left|\frac{1}{\lambda_{w}} \int_{0}^{z} \eta_{n}\left(z^{\prime}\right) d z^{\prime}\right|^{2}
\end{aligned}
$$

Clearly the computation of the bunching and radiation parameters is quite simple after the phases $\psi_{m j}$ are evaluated by the numerical solution of the pendulum equation. Their calculation involve only two additional numerical computation steps of statistical averaging and integration.

For the purpose of comparison between the coherent and incoherent radiation parameters we cite here the corresponding expression for the radiant intensity on axis at harmonic $l$ of the incoherent undulator radiation [12]:

$$
\left(\frac{d P_{U . R .}}{d \Omega}\right)_{\Theta=0}=\frac{1}{4} \sqrt{\frac{\mu_{0}}{\epsilon_{0}}} \frac{c \lambda_{w}^{2}}{l \lambda_{l}^{3}} e I_{0}\left(\frac{\bar{a}_{w}}{\gamma}\right)^{2}[J J]_{l}^{2} N_{w}
$$

We use this expression for the computation of another parameter of interest: the ratio between the radiant intensity of the superradiant radiation to the radiant intensity of the incoherent harmonic undulator radiation. This parameter determines how much will the detector reading go up in the experiment when the laser beam is turned on, and the radiation emission turns superradiant. Using $(22,23)$ we find this ratio to be

$$
\frac{\frac{d P_{S, R}}{d \Omega}}{\left.\frac{d P_{U, R} .}{d \Omega}\right|_{\Theta=0}}=\frac{1}{4} \frac{I_{0} \lambda_{w}}{e c} \frac{l}{N_{w}} r_{n}(L)
$$

Note that this ratio is proportional to to the current, since the super radiant emission is proportional to $I_{0}^{2}$ (field amplitudes add up), while the incoherent radiation is proportional to $I_{0}$ (energies add up). Note also that the experimentally measured ratio between super-radiant and incoherent emission can be enhanced by taking advantage of the temporal coherence of the super radiant emission. Installing the detector behind a narrow band-pass filter or a spectrometer of bandwidth narrower than the undulator emission 
bandwidth $-\omega_{u} /\left(N_{w}\right)$, the factor (24) is enhanced by the ratio between the undulator radiasion bandwidth to the filter bandwidth.

\section{RESULTS OF NUMERICAL COMPUTATIONS}

The extended pendulum equation solving code PENTOK, was employed to solve the set of equations (6) for a simple example of a cold electron beam with vanishing spread parameters and the experimental parameters of Table 4. The beam was modeled by 36 electrons, equispaced in their initial phases. This ample-electrons number is four times the highest order computed harmonic number $(n=9)$, as required by the Nyquist criterion. It was confirmed to provide an adequate sampling accuracy, by verifying that doubling the sample electrons number did not change the final computed parameters.

The solutions for the electron phases were used in (20b) and (22b) to compute the bunching parameter $\eta_{n}$ and the radiation parameter $r_{n}$. Their dependence on the interaction length $z$ at harmonics $(n=1,3,5,7,9)$ are displayed in Figs. 8 and 9 for beam energy $\gamma=\gamma_{\text {rad }}^{\max }=783.54$ (for $w h i c h$ the radiation parameter was maximal at $z=L_{w}=2.2 \mathrm{~m}$ ). The values of the parameters at the end of the wiggler were used to calculate the radiant intensity on axis (22a) and its ratio to the incoherent radiant intensity (24). These are listed in Table 5 for tine different harmonics.

The bunching parameters at $\gamma=\gamma_{r a d}^{\max } \approx \gamma_{R}$ (shown in Fig. 8) are somewhat lower than their.values at $\gamma=\gamma_{\text {bunch }}^{\text {max }}=785.64$ for which the phase space diagram of Fig. 3 was drawn (about $5 \%, 12 \%$, and $17 \%$ reduction for $n=1,3,5$ at the end of the wiggler). Still substantial bunching $\left(\eta_{1}=93 \%, \quad \eta_{3}=61 \%, \eta_{5}=49 \%\right)$ is attained at the lower harmonics. The big variation among the harmonics and the small efficiency of high order harmonic bunching indicate that maximum phase bunching and radiation saturation were not yet attained within the interaction length (as also evident from Fig. 4).

The big variation among the harmonics is even more pronounced when considering the radiation parameter (Fig. 9). The power law dependence on $z$, which is suggested by the linear behavior of the curves in the logarithmic scales, also indicates that over-bunching was not yet attained and substantial enhancement (especially at higher order harmonics) may be obtained in an example of a longer wiggler or a more intense modulating laser field.

The main measurable data is summarized in Table 5. The table indicates that even with a low effective (peak) current of $\left(I_{0}\right)_{e f f}=1 \mathrm{~mA}$, the super- radiant emission radiant intensity exceeds the spontaneous radiant intensity at laser harmonics as high as $n=9$ (undulator harmonic $l=27$ ). The detectability may be even further enhanced by means of a monochromator. It is, though, a little hard to "estimate the value of $\left(I_{0}\right)_{\text {eff }}$ that in 


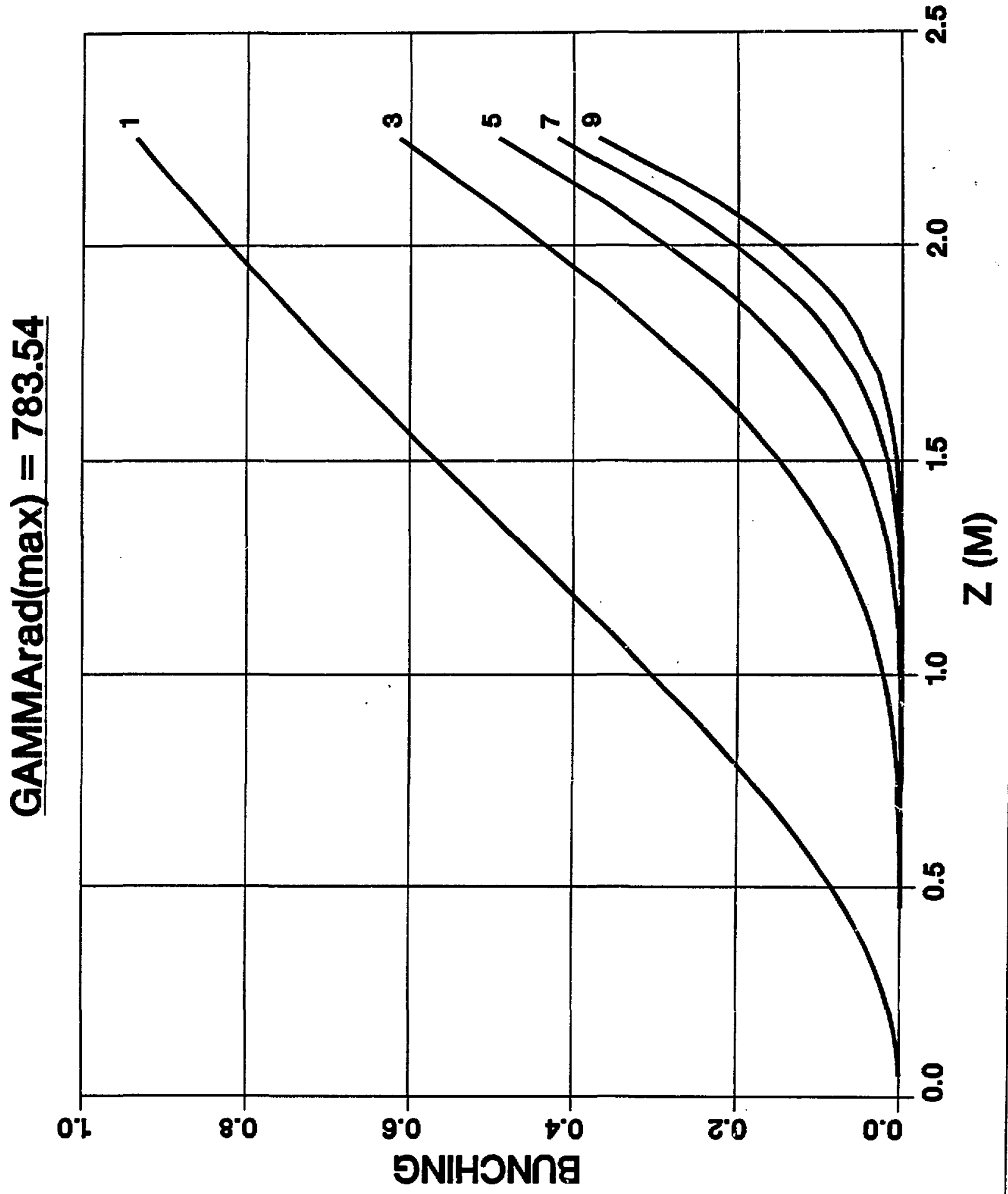

Fig. 8 The bunching parameter of various laser harmonics (n) at condition of beam energy tuning to maximum coherent radiation on axis $\gamma=\gamma_{\text {rad }}^{\max }$. 


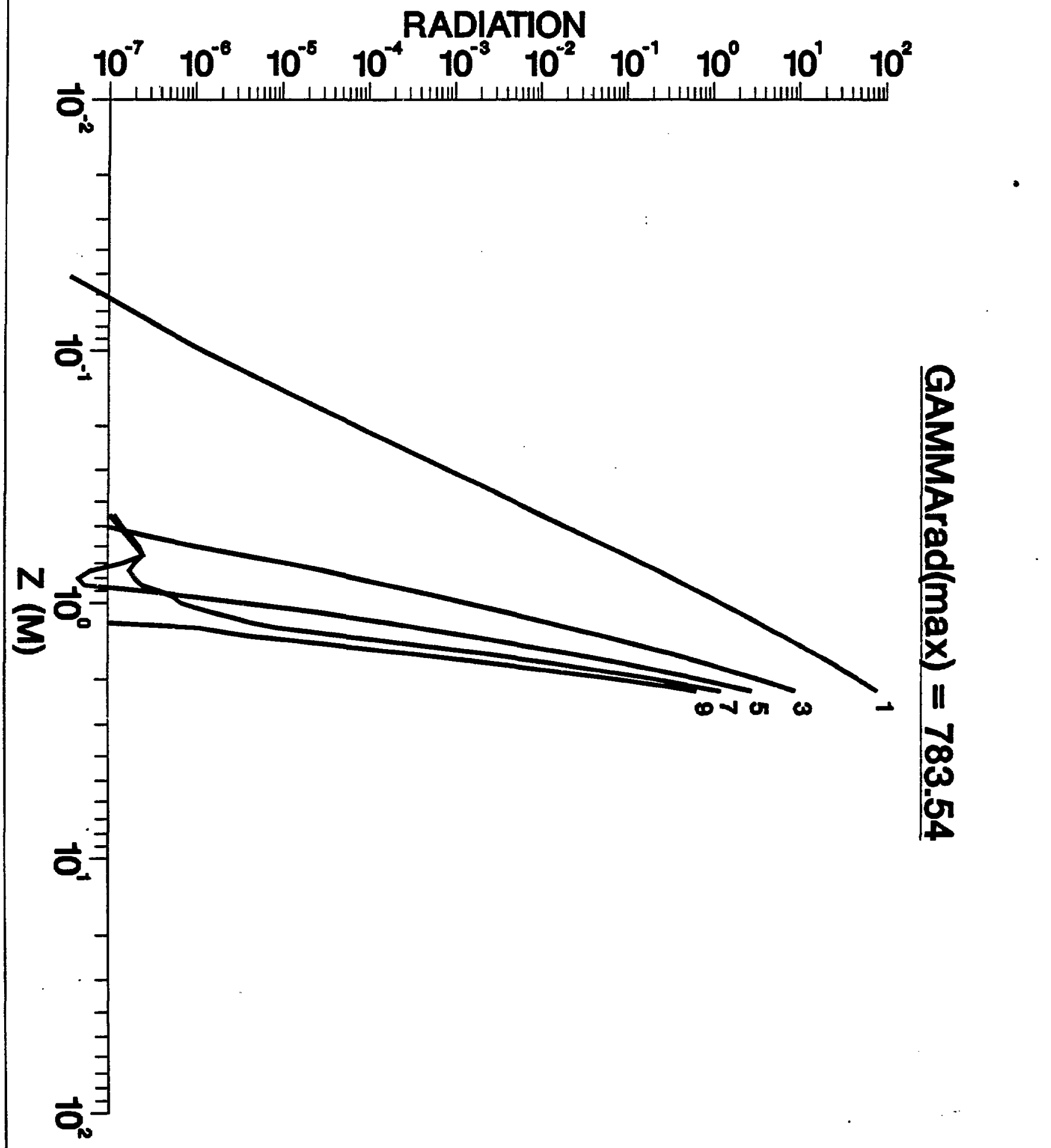


a specific experiment, will fit the simple model of the zero-spread cold-beam example of Table 4. Peak currents in storage rings may well exceed the tens of Ampers range, but only a small portion of the beam current will fit into the phase space acceptance volume for which a no-spread cold beam model can be taken.

While the simple zero-spread-cold beam example is helpful for understanding the basic features of the interaction, for a full quantitative evaluation of a super-radiant emission experiment, all the beam-spread parameters must be included in the computation. This realistic modelling was indeed implemented in the computer program, but the numerical computations were so far carried out only in part, and will not be reported yet in the present publication.

In conclusion of the discussion on the numerical results, we now explain in some more detail the 3-D effect which resulted in a maximum bunching energy $\gamma_{b u n c h}^{\max }$ appreciably larger than both the "plane wave" resonance energy $\gamma_{R}$ and the maximum radiation parameter energy $\gamma_{\text {rad }}^{\max } \approx \gamma_{R}$. The understanding of this 3-D effect has implications of the interpretation the experimentally measurable parameters of Table 5.

Inspection of Eq. (5b) for the Gaussian phase expression reveals that within the Rayleigh range region a Gaussian mode behaves like a free-space plane wave with a slightly smaller wave number (larger axial wavelength). In a first order expansion in terms of $\left|z-z_{w}\right| / z_{R}$ (assuming $z_{R_{x}}=z_{R_{y}}, \quad z_{w_{x}}=z_{w_{y}}, \quad x=y=0$ ):

$$
k_{e f f}=\frac{\omega}{c}+\frac{\partial \Phi}{\partial z} \approx \frac{\omega}{c}-\frac{1}{z_{R}} \text {. }
$$

Correspondingly also the phase velocity of the wave $v_{p h}=\omega / k_{e f f}$ is slightly faster than the speed of a free space plane wave $(c)$. Consequently, in order to maintain synchronism between the electron and the ponderomotive wave within the Rayleigh range region (where most of the energy bunching takes place) the speed of the electrons must also be increased correspondingly.

Examination of the resonance condition $k_{e f f}+m k_{w}=\omega / v_{z}$ reveals that the effect of the Gaussian wave effective wave number depreciation $-1 / z_{R}(25)$ is the same as decreasing the wiggler harmonic wave number, $m k_{w}$, ard it leads likewise to a relative increase in the resonance energy:

$$
\frac{\Delta \gamma}{\gamma_{R}}=\frac{1}{2 m} \frac{\lambda_{w}}{z_{R}}
$$

This equation results in $\Delta \gamma / \gamma_{R}=5.3 \times 10^{-3}$ with the parameters of Table 4. This agrees reasonably well with the results of the numerical computations which gave $\left(\gamma_{b u n c h}^{\max }-\right.$ $\left.\gamma_{R}\right) / \gamma_{R}=3.2 \times 10^{-3}$, in support of our interpretation.

To understand why $\gamma_{\text {rad }}^{\max } \neq \gamma_{\text {bunch }}^{\max }$ we draw attention to the fact that the strong bunching effect which takes place at $\gamma=\gamma_{b u n c h}^{\max }$ is not very helpful for efficient radiation into a 
plane wave on axis (which is the parameter measured in (22)). The reason is that the energy bunching with the "fast" Gaussian wave $\left(k_{e f f}<\omega / c\right)$ produces towards the end of the wiggler a polarization current with a correspondingly reduced wave number (higher phase velocity). This current cannot excite efficiently a coherent harmonic frequency radiation plane wave that propagates on axis at the speed of light $c$ with free space wave number $\omega_{n} / c$. To maximize such an emission, it turned out to be more favorable to operate at $\gamma=\gamma_{\text {rad }}^{\max } \approx \gamma_{R}$ so that synchronization between the electrons and the modulating laser beam is attained away from the Rayleigh range region, where the Gaussian phase shift diminishes, and it behaves more like a a free space plane wave. The numerical simulation results indicate that it is preferable to operate off the maximum bunching condition $\gamma=\gamma_{\text {bunch }}^{\max }$ despite the advantage of efficient energy modulation by the strong field at the Gaussian waist region, because one loses then more by the bunching of the electrons at the wrong wave number, which results in destructive interference of the generated radiation, and consequently reduce the coherent emission on axis.

A number of practical conclusions may be derived from this interpretation. Some simple remedies can be devices in order to enhance the super-radiant emission radiant intensity on axis, if this is the desirable parameter. In the first place, it seems to be preferable to focus the laser beam waste at a more forward (up stream) point, so that near plane wave energy modulation can take place from the start. Another scheme can be to taper the wiggler period towards the end of the wiggler, in order to phase-match the polarization current with a desirable on-axis harmonic frequency plane wave and still operate at the conditions of maximum bunching $\gamma \approx \gamma_{b u n c h}^{\max }$. We note, however, that the maximization of the radiant intensity is not always the main experimental goal, and if other parameters are important, the operative conclusions may be different.

It can be well appreciated that if the wave number of the harmonic frequency polarization current is too small to phase match well to a forward going radiation plane-wave, it can still match well to an off-axis wave (of axial wave number $\left(\omega_{n} / c\right) \cos \Theta$ ). This may lead to a conclusion that at $\gamma=\gamma_{\text {bunch }}^{\max }$ the total coherent harmonic radiation in all angles is still high, even though the emission on axis is reduced (relative to a gamma $\approx \gamma_{R}$ tuning condition). Since the emission from a zero width current source is spatially coherent in either case, we may conclude that not only the total power but also the brightness of the radiation could be larger at $\gamma=\gamma_{\text {bunch }}^{\max }$. Since the radiation is still spatially coherent, its larger divergence is not necessarily a deficiency if optics is available to collimate or focus it to a desirable beam spot.

We note in conclusion that the observations we have just made, may be modified when the coherent radiation is emitted from a beam of a finite width which is larger than the 
effective coherence width of the zero beam-width super radiant harmonic emitter. In this latter case, the diffraction characteristics may be determined by an effective aperture which depends on the electron beam size. We conclude that substantial and non-negligible 3-D effects are expected in the analysis of the super-radiant emission inieraction, and that these effects can be studied in the framework of the physical model and computer program we developed. We expect to report further numerical computation results on the effects of e-beam spread parameters in a future publication.

\section{REFERENCE}

1. G. Vignola, R.R. Freeman, B.M. Kincaid, C. Pellegrini, A. Luccio, J. Murphy, J. Galayda and A. Van-Steenbergen, Nucl. Inst. and Meth. in phys. Res. A239 43 (1985).

2. B. Girard, Y. Lapierre, J.M. Ortega, C. Bazin, M. Billardon, P. Elleaume, M. Bergher, M. Velghe, Y. Petroff, Phys. Lett. 53, 2405 (1984)

3. S. Benson, "Status of the Mark III FEL Project", in this proceeding.

4. G.R. Neil, J.A. Edighoffer, S.W. Fornaca, C.E. Hess, T.I. Smith and H.A. Schwettman, Proceeding of the 1984 FEL Conference, Castelgandolfo (Rome), Italy, Sept. $1984 \mathrm{p}$. 199.

5. I. Schnitzer and A. Gover, Nucl. Inst. and Meth. in Phys. Res. A237,124 (1985)

6. A. Gover, H. Freund, F.L. Granatstein, J.H. McAdoo, C.M. Tang, Infrared and Millimeter Waves, 11, 291 (K. Button ed.) Academic 1984.

7. A. Gover, A. Luccio, A. Friedman and A.M. Fauchet, A.I.P. Proc. No. 147, "Short Wavelength Coherent Radiation: Generation and Application", Monterey, CA 1986, p. 219.

8. N.S. Ginzburg and M.D. Tokman, Sov. Phys. Tech. Phys. 29 (6), 604 (1984).

9. C.W. Gear, "Nusnerical Initial Value Problems in Ordinary Differential Equations", Prentice-Hall, Englewood Cliffs, New Jeresey, 1971.

10. M.J. Bastiaans, J. Opt. Soc. Am. 69, 1710 (1979).

1i. K.J. Kim, "Brightness, Coherence and Propagation Characteristics of Synchrotron Radiation", Lawrence Berkeley Laí. Report No. LBL-20181 July 1985.

12. S. Krinsky, W. Thomlinson, A. Van-Steenbergen, Informal Report No. BNL 3198S, Sept. 1982. 
Table 1: Classical Definitions of Padiation Schemes

(1) UNDULATOR SYNCHROTRON RADIATION

(FEL SPONTANEOUS EMISSION): The temporally incoherent radiation of electron entering into a wiggler/undulator at random.

(2) SUPERRADIANT FREE ELECTRON RADIATION: the temporally coherent radiation of bunched electrons oscillating in a wiggler/undulator in phase with each other.

(3) FREE ELECTRON LASING: The coherent emission of bunched electrons radiating in phase into the same frequency and spatial domain of the radiation field which produced the bunching.

Table 2: Frequencies Involved

\begin{tabular}{|c|c|c|}
\hline Frequency & Notation/Definition & Linewidth \\
\hline Fundamental undulator frequency & $\omega_{u}=\frac{k_{u} c}{\theta^{-1}-1} \approx 2 \gamma_{z}^{2} k_{w} c$ & $\frac{\Delta \omega_{u}}{\omega_{u}}$ \\
\hline Modulating laser frequency & $\omega_{m} \approx m \omega_{u}$ & $\frac{\Delta \omega_{m}}{\omega_{m}}=\frac{2 \pi / T_{\text {laser }}}{\omega_{m}}$ \\
\hline n-th order coherent harmonic & $\omega_{n}=n \omega_{m}\left(\approx n m \omega_{u}\right)$ & $\frac{\Delta \omega_{n}}{\omega_{n}}=\frac{2 \pi / T_{\text {pulse }}}{\omega_{n}}$ \\
\hline$l$-th order incoherent harmonic of wiggler & $\omega_{u l}=l \omega_{u}$ & $\frac{\Delta \omega_{u l}}{\omega_{u l}}=\frac{l}{l N_{w}}$ \\
\hline
\end{tabular}

\section{Table 3: 3-D Code Model Features}

(1) Initial spatial spread $\left(\sigma_{x}, \sigma_{y}\right)$.

(2) Initial angular spread $\left(\sigma_{x^{\prime}}, \sigma_{y^{\prime}}, z_{e w x}, z_{e w y}\right)$.

(3) Initial energy spread \& tuning $\left(\sigma_{\gamma}, \gamma_{0}\right)$.

(4) General modulating mode $\left(w_{0 x}, z_{w x}, w_{0 y}, z_{w y}\right)$.

(5) Excited radiation described in terms of discrete modes (Hermit Gaussian) / plane waves (angles).

(6) Optical parameters: Power/radiant intensity/brightness and the corresponding spectral parameters.

(7) Possible extension: Magnet field variation and error.

Table 4: No Beam-Spread Example

\begin{tabular}{|ll|}
\hline$\lambda_{w}=10 \mathrm{~cm}$ & $z_{w}=0.3 \mathrm{~m}$ \\
$N_{w}=22\left(L_{w}=2.2 \mathrm{~m}\right)$ & $P_{m}=90 M W a t t$ \\
$\bar{a}_{w}=4.31$ & $m=3$ \\
$\lambda_{m}=0.5320 \mu m$ & $n=1,3,5,7,9 \quad(l=3,9,15,21,27)$ \\
$z_{R}=0.5 m$ & No angular, spatial and energy spread \\
\hline
\end{tabular}




\begin{tabular}{|cc|c|c|c|}
\hline \multicolumn{2}{|c|}{ Harmonic } \\
$n$ & $(l)$ & $\begin{array}{c}\text { Wavelength } \\
(\AA)\end{array}$ & $\begin{array}{c}\frac{d P}{d \Omega} \\
{[W / \text { Ster }]}\end{array}$ & $\frac{d P_{S . R .} . d \Omega}{d P_{U . R .} / d \Omega}$ \\
\hline 1 & $(3)$ & 5320 & $1.938 \times 10^{8} I_{0}^{2}$ & $1.815 \times 10^{16} I_{0}$ \\
3 & $(9)$ & 1773 & $3.905 \times 10^{7} I_{0}^{2}$ & $1.058 \times 10^{14} I_{0}$ \\
5 & $(15)$ & 1064 & $1.526 \times 10^{7} I_{0}^{2}$ & $2.363 \times 10^{15} I_{0}$ \\
7 & $(21)$ & 760 & $7.507 \times 10^{6} I_{0}^{2}$ & $3.964 \times 10^{15} I_{0}$ \\
9 & $(27)$ & 591 & $4.182 \times 10^{6} I_{0}^{2}$ & $5.736 \times 10^{15} I_{0}$ \\
\hline
\end{tabular}

\section{Figure Captions}

Fig. 1 Schemes for super-radiant (bunched beam) coherent harmonic generation.

Fig. 2 Axial and transverse cross-sectional schematics of the electron beam and the modulating radiation beam.

Fig. 3 Energy-phase-space diagram of electrons at four different points along the wiggler for beam energy tuning to maximum phase bunching $\gamma=\gamma_{\text {bunch }}^{\max }$.

Fig. 4 Continuous Energy-phase-space diagrams of electron trajectories along the entire wiggler for beam energy tuning to plan wave resonance condition $\gamma=\gamma_{R}$.

Fig. 5 Mechanical analogue diagram of the Gaussian phase "jolt effect". Only the highly stable and unstable particles experience reversal of force direction throughout the process.

Fig. 6 Schematic illustration of wave packet emission into a transverse radiation mode by single electrons. The diagram depicts an example of a four period wiggler and corresponding four period optical wavepacket.

Fig. 7 Time-frequency phase space picture of spontaneous and super-radiant emission into a transverse radiation mode.

Fig. 8 The bunching parameter of various laser harmonics ( $n$ ) at condition of beam energy tuning to maximum coherent radiation on axis $\gamma=\gamma_{\text {rad }}^{\max }$.

Fig. 9 The on axis radiation parameter of various harmonics corresponding to the parameters of Fig. 8.

\section{DISCLAIMER}

This report was prepared as an account of work sponsored by an agency of the United States Government. Neither the United States Government nor any agency thereof, nor any of their employees, makes any warranty, express or implied, or assumes any legal liability or responsibility for the accuracy, completeness, or usefulness of any information, apparatus, product, or process disclosed, or represents that its use would not infringe privately owned rights. Reference herein to any specific commercial product, process, or service by trade name, trademark, manufacturer, or otherwise does not necessarily constitute or imply its endorsement, recommendation, or favoring by the United States Government or any agency thereof. The views and opinions of authors expressed herein do not necessarily state or reflect those of the United States Government or any agency thereof. 